\title{
Comprehensive Data Scientific Procedure for Enhanced Analysis and Interpretation of Real-Time Breath Measurements in In Vivo Aroma- Release Studies
}

Ewa Szymańska, ${ }^{* \dagger, \ddagger}$ Phil A. Brown, ${ }^{\dagger, \ddagger}$ Aldo Ziere, ${ }^{\S}$ Sara Martins, ${ }^{\S}$ Max Batenburg, ${ }^{\S}$ Frans J. M. Harren, and Lutgarde M. C. Buydens

${ }^{\dagger}$ TI-COAST, P.O. Box 18, 6160 MD Geleen, The Netherlands

${ }^{\ddagger}$ Radboud University Nijmegen, Institute for Molecules and Materials (IMM), P.O. Box 9010, 6500 GL Nijmegen, The Netherlands

${ }^{\S}$ Unilever R\&D, P.O. Box 114, 3130 AC Vlaardingen, The Netherlands

Supporting Information

ABSTRACT: Real-time measurements of many low-abundance volatile organic compounds (VOCs) in breath and air samples are already feasible due to progress in analytical technologies, such as proton transfer reaction mass spectrometry (PTR-MS). Nevertheless, the information content of real-time measurements is not fully exploited, due to the lack of suitable data handling methods. This study develops a data scientific procedure to enhance data analysis and interpretation of longitudinal, multivariate data sets from real-time, in vivo, aroma-release studies. The developed procedure includes an automated data preprocessing and a multivariate assessment of the test panel performance. A large multifactorial PTR-MS data set is investigated that includes four experimental protocols, two tested food products, four aroma

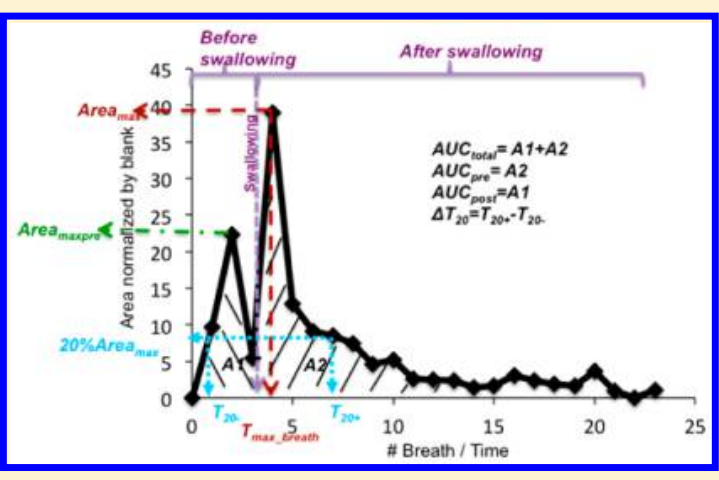
compounds, and eight panelists. Real-time measurements are converted into standardized breath profiles by preprocessing, and 10 kinetic parameters are derived. Next to this, panel performance is evaluated per experimental protocol and food product. Comprehensive information about panel performance, individual panelists, studied products, aroma compounds, and kinetic parameters is extracted, demonstrating the great value of the developed approach.

$\mathrm{R}$ eal-time measurements of many low-abundance volatile organic compounds (VOCs) in breath and air samples are currently feasible by using, for example, proton transfer reaction mass spectrometry (PTR-MS) ${ }^{1-4}$ and atmospheric pressure chemical ionization source mass spectrometry (APCI-MS).$^{5-8}$ These measurements can be used in different fields including:

(a) clinical applications, such as online monitoring of (patho)physiologically induced breath biomarker profiles $^{2}$ and pharmacokinetics investigations of drugs eliminated or absorbed by the respiratory tract ${ }^{9}$

(b) process analysis: for example, online monitoring of VOCs in off-line gases of bioreactors ${ }^{10}$

(c) food and nutritional applications: for example, in online in vivo retronasal flavor release analysis ${ }^{6}$

By analyzing concentrations of VOCs over time, new insights could be gained. These insights are not only about dynamics of the processes involved but also about the effect of different perturbations on these processes, such as the effect of the food matrix on aroma release.

Real-time measurements provide longitudinal, multivariate data sets, which contain information about time courses of the monitored VOCs. Longitudinal multivariate data sets can be analyzed directly with various approaches ${ }^{11}$ or indirectly by deriving a plethora of kinetic parameters. Kinetic parameters such as the maximum intensity time and the half-life time are commonly used in pharmacokinetic and nutrikinetic studies. ${ }^{9,12,13}$ However, currently used procedures for data analysis and interpretation of real-time VOC profiles are very limited, especially in breath analysis. In such studies, exhaled air from spontaneously breathing patients or volunteers is analyzed. This yields real-time profiles with a breathing pattern that is variable in frequency and intensity between volunteers and experimental conditions; this significantly hampers analysis of real-time breath profiles.

At present, no procedure for synchronization and standardization of real-time breath measurements is available. Current data scientific procedures are confined to directly calculating or reading kinetic parameters such as $C_{\max }$ (maximum concentration), $T_{\max }$ (time of maximum concentration), and AUC (area under curve in a plot concentrations vs time) from real-time profiles, ${ }^{7,14}$ collecting maximum concentrations from the few selected breaths ${ }^{15}$ or smoothing of real-

Received: June 19, 2015

Accepted: September 23, 2015

Published: September 23, 2015 
time profiles to obtain estimations of time trajectories. ${ }^{16,17}$ These methods provide a limited description of time courses. The description allows only a basic interpretation of breath profiles, for example, by standard univariate tests, such as analysis of variance (ANOVA).

The aim of this paper is to develop a comprehensive data scientific procedure to enhance data analysis and interpretation of real-time measurements from in vivo, aroma-release studies. The procedure includes both an automated data preprocessing of real-time breath measurements and a multivariate assessment of derived kinetic parameters.

The main steps of the procedure are described in the Procedure section, and experimental settings are given in the Methods section. The procedure was successfully tested on a large data set measured by PTR-MS in an in vivo aroma-release study (see Materials). This data set was collected to select an experimental protocol with optimal panel performance. Application of the developed procedure is described in the Results section. It includes standardization of time profiles, derivatization of 10 kinetic parameters, and comprehensive evaluation of panel performance in different protocols. Panel performance was assessed by considering the repeatability of the panel and of individual panelists as well as the discrimination power of the panel to distinguish between the two tested food products. Multivariate methods were introduced and applied for this purpose, the first time such methods are applied to in vivo flavor-release studies. Advantages of our procedure in reference to currently used strategies are apparent (see Discussion. A comprehensive interpretation of differences between experimental protocols in the analyzed study is provided and discussed in the Discussion section.

\section{PROCEDURE}

The first part of the developed procedure involves an automated data preprocessing which consists of:

1. baseline correction,

2. detection of the starting and ending points of each breath stroke for a given reference ion,

3. transformation of real-time measurements into breathby-breath profiles,

4. standardization of breath-by-breath profiles,

5. derivation of kinetic parameters.

These steps are presented in Figure 1. Implemented methods are briefly described below and in detail in the Methods section.

In Figure 1A, the real-time measurements of acetone (a reference ion; a ubiquitous, abundant compound in breath ${ }^{18}$ ) and one of the monitored ions (ethyl octanoate) are shown. Start, product, and swallow time are displayed. Clear breath pattern, with regular breath strokes, is visible for acetone, and a less regular pattern is observed for ethyl octanoate.

During preprocessing in step 1 , any drift of the baseline is corrected. The aim of step 2 is to extract the breath pattern of acetone to use it in step 3. The starting and ending points of each breath stroke for a given reference ion are detected by minima detection (Figure 1B). Starting and ending points corresponding to thin peaks (i.e., not breath strokes) with areas smaller than $25 \%$ are not included in step 3 .

In step 3, the real-time measurements are transformed into breath-by-breath profiles in order to eliminate differences of breath pattern frequency and intensity between volunteers and experimental conditions. Breath areas from the real-time

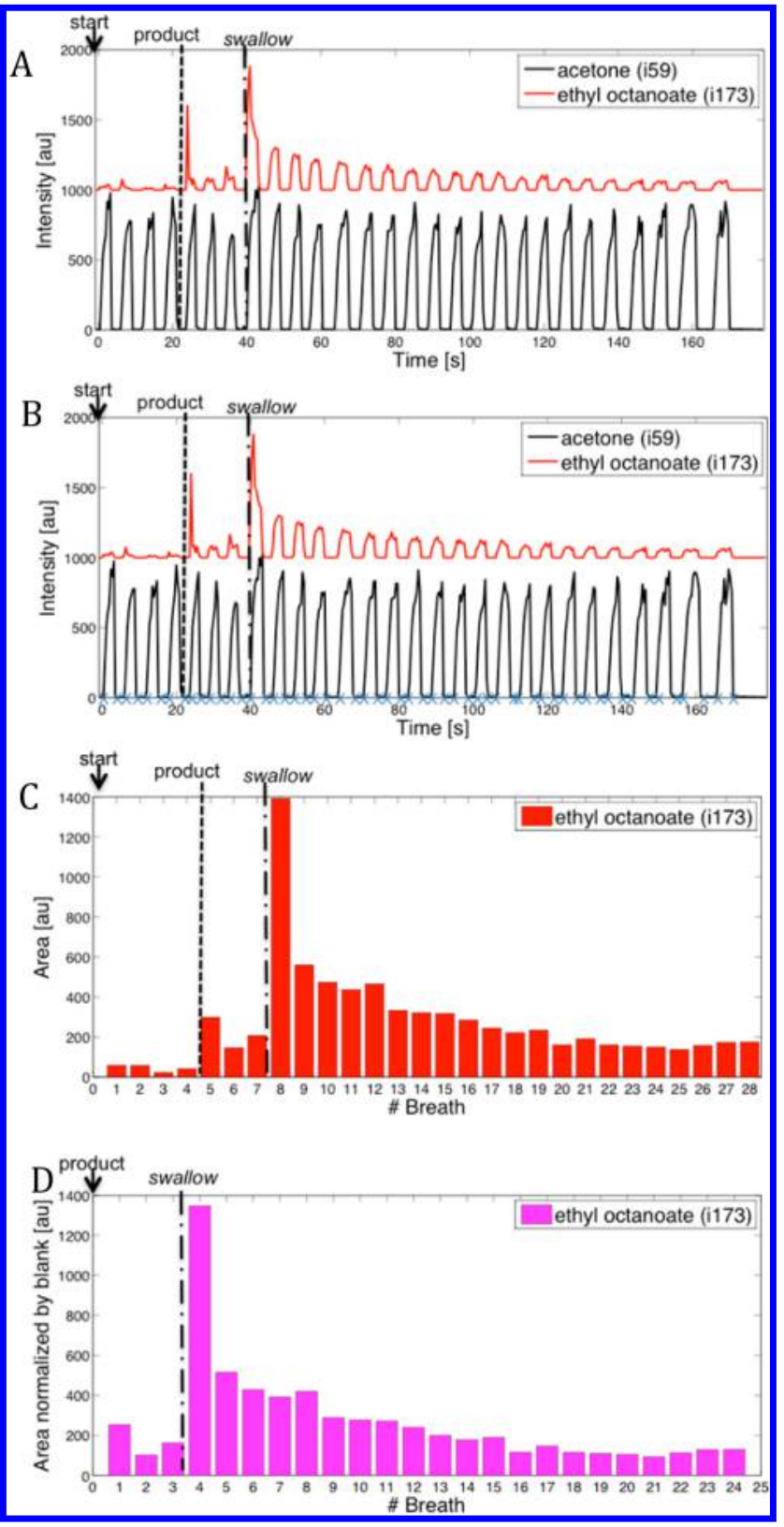

Figure 1. Transformation of real-time measurements into standardized breath-by-breath profiles during developed preprocessing procedure. Panel A: original real-time measurements for two compounds: acetone (reference ion) and ethyl octanoate (monitored ion) for panelist $\mathrm{p} 1$, following protocol A and processing product $f f$. Start, product, and swallow times are indicated. Panel B: real-time measurements after baseline correction and detection of the starting and ending points of the breath strokes for the acetone. Panel C: breath-by-breath profiles of ethyl octanoate calculated with the trapezoidal numerical integration method, using the start and end points from the previous step (B). Panel D: breath-by-breath profiles of ethyl octanoate after administering the product.

measurements of ethyl octanoate are calculated with a trapezoidal numerical integration method for each breath using the detected starting and ending points of each breath stroke from step 2. Breath-by-breath profiles are obtained by sorting breath areas according to their order in time i.e. first, second, third, ..., last collected breath area (Figure 1C).

In step 4, standardization of ethyl octanoate breath-by-breath profiles takes place. The developed procedure consists of both 
normalization to acetone breath-by-breath profiles and correction for background levels of ethyl octanoate in breath before product administration. The normalization to acetone breath-by-breath profiles was not performed in this study. Breath-by-breath profiles of ethyl octanoate are standardized only by background levels of ethyl octanoate in breath before product administration i.e. the blank period (step 4, Figure 1D). The breath profile of the product period is corrected by subtracting the mean breath area of the blank period from each breath area from the product period. Before standardization, breaths in the blank period are checked for outliers (i.e., any breaths with areas larger than twice the mean breath area of the first two breaths in the product period). This rule was optimized and corresponds well to the outlier detection with visual inspection. Outliers are eliminated.

In the final step of preprocessing, 10 kinetic parameters describing the release kinetics of each compound are derived (Figure 2 and Table 2). These parameters are derived either from standardized breath-by-breath profiles or from real-time measurements after baseline correction as described in Methods.

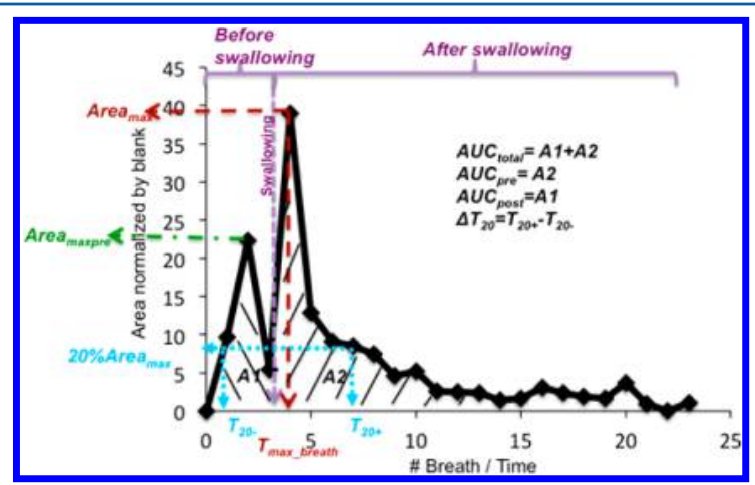

Figure 2. Deriving kinetic parameters: total area under the curve $\left(\mathrm{AUC}_{\text {total }}\right)$, maximum breath area $\left(\mathrm{Area}_{\max }\right)$, area under the curve before swallow $\left(\mathrm{AUC}_{\mathrm{pre}}\right)$, maximum breath area before swallow $\left(\right.$ Area $\left._{\max \text { pre }}\right)$, area under the curve after swallow $\left(A U C_{\text {post }}\right)$, time required to reach $20 \%$ of maximum breath area after time of maximum breath area $\left(T_{20+}\right)$, time required to reach $20 \%$ of maximum breath area before time of maximum breath area $\left(T_{20-}\right)$, difference between $T_{20+}$ and $T_{20-}\left(\Delta T_{20}\right)$. Time of maximum intensity $\left(T_{\max }\right)$ and maximum intensity $\left(C_{\max }\right)$ are read from real-time measurement after baseline correction.

After preprocessing of all real-time measurements of a considered data set (see Materials), the preprocessed data set includes 2 food products, 4 protocols, 8 panelists, 5 repetitions, 4 compounds, and 10 kinetic parameters.

In the second part of the developed procedure, the preprocessed data set is used to evaluate panel performance in each of the four considered experimental protocols. The repeatability of each individual panelist and the panel as well as discriminant power of the panel are evaluated. Univariate and multivariate methods are employed (see Methods).

The repeatability of each individual panelist is assessed with a measure adapted from Rossi. ${ }^{19}$ The repeatability of the panel in each protocol is assessed by means of the pooled variance over all panelists. Both repeatability measures are calculated for each protocol, product, ion, and kinetic parameter combination.

First, the panel repeatability is evaluated with standard univariate tests (ANOVA and Wilcoxon tests) separately for every ion, kinetic parameter, and product combination. Second, individual panelist repeatabilities of all ion and parameter combinations are included in principal component analysis (PCA). For between protocol comparisons, PCA is performed on data from all protocols. To explore differences within each protocol, PCA is performed per protocol.

To evaluate discrimination power of each experimental protocol, differences between two products are assessed with standard univariate tests (Wilcoxon tests) separately for every ion, kinetic parameter and protocol combination. Moreover, multilevel partial least squares discriminant analysis (ML-PLSDA) is employed. Four ML-PLS-DA models are built, one for each protocol. They include kinetic parameter levels for all ion and parameter combinations. The discrimination power of the protocols is assessed comparing performance measures of MLPLS-DA models.

Finally, outcomes of univariate and multivariate evaluation are compared and combined. This provides an enhanced interpretation of differences in panel performance. Complementary information about individual panelists, studied products, aroma compounds and kinetic parameters, is extracted, demonstrating the strength of the developed procedure.

\section{EXPERIMENTAL SECTION}

Materials. Products and Their Preparation. Two products are tested: product $f f$ (full fat milk) and ss (aqueous sugar solution). They both contain four aroma compounds: acetoin, anisaldehyde, ethyl butyrate, and ethyl octanoate (see Table 1) at a concentration of $135,137,93$, and 135 ppm, respectively. These compounds are aroma compounds commonly present in food products and are able to be detected by PTR-MS. They were specifically selected in this study to span two groups of aroma compounds: esters and aldehydes and compounds with a wide range of physiochemical properties such as $\log P$ (partition coefficient between oil and water, a measure of lipophilicity) and $K_{\mathrm{a} / \mathrm{w}}$ (partition coefficient between air and water, a measure of volatility).

Table 1. Aromatic Compounds and Monitored Ions

\begin{tabular}{|c|c|c|c|c|c|c|}
\hline compound & CAS & molecular weight/Da & ion monitored in PTR-MS $\mathrm{m} / \mathrm{z}$ & $\log P^{b}$ & $K_{\mathrm{a} / \mathrm{w}}{ }^{c}$ & odor description \\
\hline acetoin & $513-86-0$ & 88.11 & $87^{a}$ & -0.32 & $4.21 \times 10^{-4}$ & buttery, pastry \\
\hline ethyl butyrate & $105-54-4$ & 116.16 & 117 & 1.85 & $1.63 \times 10^{-2}$ & fruity, orange, pineapple \\
\hline anisaldehyde & $123-11-5$ & 136.15 & 137 & 1.76 & $3.25 \times 10^{-5}$ & anise, sambuca, ouzo \\
\hline ethyl octanoate & $106-32-1$ & 172.27 & 173 & 3.81 & $5.19 \times 10^{-2}$ & fruity \\
\hline
\end{tabular}

${ }^{a}$ PTR-MS typically allows product ions to be monitored at the molecular mass of the compound $+1 \mathrm{Da}$. In the case of acetoin, fragmentation and loss of $\mathrm{H}_{2}$ occurs, and thus, the product ion is detected at $-1 \mathrm{Da} .{ }^{b} \log P-$ partition coefficient between oil and water, a measure of lipophilicity ${ }^{c} K_{\mathrm{a} / \mathrm{w}}$ - partition coefficient between air and water, a measure of volatility 
Product $f f$ (full fat milk) has an addition of sugar at $3 \%$ concentration. Product ss has a $10 \%$ sugar solution. Please see Supporting Information for more details.

Panelists. Eight trained panelists $(\mathrm{p} 1-\mathrm{p} 8)$ are included in this study comprising 4 female and 4 male volunteers, aged 1865.

Protocols. Four experimental protocols (A, B, C and D) are tested in this study. A single assay with each protocol lasts for 2 min. First, for a duration of $20 \mathrm{~s}$, room air is sampled, after which panelists position the sample device (see below) in their nostrils. The panelists are asked to take four regular breaths and then to administer the product, as indicated in Figure 1A. The time of starting the PTR-MS measurement (start), the time at which the product is administered (product), and the time of the first swallow and other swallows (swallow times) are recorded for each assay. A $5 \mathrm{~mL}$, plastic syringe is used by each panelist to administer $5 \mathrm{~mL}$ of the sample in the mouth.

Protocol A is a reference protocol. During this protocol, panelists have a free breathing pattern and swallow the ingested product only once, according to their preference. In protocol B, panelists also have a freely chosen breathing pattern but are instructed to swallow $20 \mathrm{~s}$ after the product is introduced. During protocol $\mathrm{C}$, the breathing pattern of each panelist is synchronized to a regularly beating, external sound ( 72 beats per minute). Each panelist swallows once every $20 \mathrm{~s}$ after administering the product. In protocol $\mathrm{D}$, panelists have a freely chosen breathing pattern and swallow four times: first, $20 \mathrm{~s}$ after the product was introduced and every $20 \mathrm{~s}$, thereafter.

The panelists received instructions beforehand and had time to practice the protocols and familiarize themselves with the equipment. Between each assay, panelists are asked to cleanse their mouth, rinsing with water. For each protocol, product, and panelist, assays were repeated five times. That lead to 320 assays measured by the PTR-MS equipment. Due to an error in data collection, two data files were incorrectly saved, giving 318 assays to be included in this study.

Methods. Breath Volatile Measurement by PTR-MS. The instrument used for measurement was a high sensitivity PTRQMS coupled with a specialized inlet system for nose-space analysis (Ionicon Analytick GmbH, Innsbruck, Austria). Samples were measured with a multiple ion detection (MID) setting to observe ions at $m / z 21,37,59,87,117,137$, and 173, and $m / z 21$ and 37 correspond to reagent ions in PTR-MS; $m$ / $z 59$ is the product ion of acetone. Other $\mathrm{m} / z$ values monitored relate to the aroma compounds, as indicated in Table 1. Please see Supporting Information for more details.

Baseline Correction. Assymetric Least Squares (ALS $)^{20}$ with the Whittaker smoother and baseline parameters $(p=10$ and $\lambda$ $=0.0001)$ were used to correct for the baseline of real-time measurements.

Detection of Breath Peak Minima in a Reference Ion Profile. Extrema function ${ }^{21}$ was used to detect minima corresponding to the starting and ending time points of breath strokes.

Deriving Kinetic Parameters. The maximum intensity in the product period $\left(C_{\max }\right)$ and the time when this occurs $\left(T_{\max }\right)$ are obtained from the real-time measurements after baseline correction. Areas under the curve $\left(\mathrm{AUC}_{\text {total }}, \mathrm{AUC}_{\mathrm{pre}}\right.$ and $\mathrm{AUC}_{\text {post }}$ ) are obtained by summing the breath areas in the total product period and the before and after swallowing periods, respectively. By selecting the maximum breath areas in a product period or before swallow period, maximum breath areas (i.e., Area $\max _{\max }$ and $\mathrm{Area}_{\max \_ \text {pre }}$ ) are collected, respectively. The two time points when $20 \%$ of Area $_{\max }$ are reached (i.e., before and after $\left.T_{\max \text { breath }}\right)$ are recorded as $T_{20-}$ and $T_{20+}$, respectively, ${ }^{22}$ where the times are recorded as the mean time of the relevant breath. Kinetic parameter $\Delta T_{20}$ is calculated as the time difference between $T_{20-}$ and $T_{20+}$.

Individual Panelist Repeatability. The repeatability of each individual panelist is a measure of repeatability provided by Mandel $^{23}$ and adapted by Rossi ${ }^{19}$ eq 1 :

$$
\text { individual panelist repeatability }=\frac{S_{i j}}{S j}
$$

where $S_{i j}$ is the standard deviation of the repetitions (in most cases 5) from each panelist calculated separately for each combination of protocol, product, ion, and kinetic parameter; $S_{j}$ is the pooled standard deviation over all panelists calculated separately for each combination of protocol, product, ion, and kinetic parameter. The closer the value of repeatability to 0 , the more consistent a panelist is relative to the overall group.

Univariate Evaluation of Panel Performance. ANOVA models and Wilcoxon signed rank tests $(\alpha=0.05)$ are used. False discovery rate correction was performed by the Benjamini and Hochberg method ${ }^{24}$ with a $q$-value $=0.05$. More details on performed analysis can be found in Supporting Information.

Multivariate Evaluation of Panel Performance. PCA is performed on individual panelist repeatabilities separately for each product. The data is autoscaled before PCA. To explore differences within each protocol, PCA is performed per protocol. It includes repeatabilities of panelists and ions in a sample mode $(n=32)$ and kinetic parameters in a variables mode $(p=10)$. For between protocol comparisons, PCA is performed on data from all protocols. It consists of repeatabilities of panelists and ions in a sample mode $(n=$ 32 ) and kinetic parameters with protocols in a variables mode $(p=40)$.

ML-PLS-DA models are double cross validated and include variable selection. ${ }^{25,26}$ In a 7 -fold double cross validated $(2 \mathrm{CV}$, external loop), all samples of the same panelist were taken aside as a test set ( 8 or 10 samples for a panelist), and the rest (a rest set) is used in the model optimization in $1 \mathrm{CV}$ (internal loop). During 5-fold single cross validation (1CV), the optimal number of latent variables and an optimal set of variables (5step variable selection) are chosen. The performance of the model is evaluated on the basis of performance parameters: the area under the receiver operating curve (AUROC) and percentage of misclassified samples (\%NMC) ${ }^{27}$ derived from predictions of the test samples in $2 \mathrm{CV}$. The statistical significance of the model performance and the selected variables is assessed with 2000 permutations.

Software. All statistical analyses are performed using Matlab (version 2014a, The MathWorks, Natick, Massachusetts, U.S.A.). The statistical toolbox of Matlab and house-written code (available on request) is used.

Hardware. The computer used in this study was Intel Xeon CPU E5-1620 3.6 GHz, 64GB RAM.

\section{RESULTS}

Data Preprocessing Procedure at Work. As an example for the data preprocessing procedure Figure 1A shows real-time profiles of acetone (a reference ion, $m / z$ 59) and ethyl octanoate $(\mathrm{m} / z$ 173). The presented data was collected from panelist p1 using product $f f$ performing protocol A. Similar breath patterns are detected for other monitored ions 
Table 2. Mean Levels and Standard Deviations of Kinetic Parameters Derived from Preprocessed Data of Four Monitored Compounds in Two Products: $f f$ and $s s$

\begin{tabular}{|c|c|c|c|c|c|c|c|c|c|c|c|c|c|c|c|c|c|}
\hline & & \multicolumn{4}{|c|}{ acetoin } & \multicolumn{4}{|c|}{ ethyl butyrate } & \multicolumn{4}{|c|}{ anisaldehyde } & \multicolumn{4}{|c|}{ ethyl octanoate } \\
\hline & & \multicolumn{2}{|c|}{ product $f f$} & \multicolumn{2}{|c|}{ product $s s$} & \multicolumn{2}{|c|}{ product $f f$} & \multicolumn{2}{|c|}{ product $s s$} & \multicolumn{2}{|c|}{ product $f f$} & \multicolumn{2}{|c|}{ product $s s$} & \multicolumn{2}{|c|}{ product $f f$} & \multicolumn{2}{|c|}{ product $s s$} \\
\hline \multicolumn{2}{|c|}{ kinetic parameter } & mean & SD & mean & SD & mean & SD & mean & SD & mean & $\mathrm{SD}$ & mean & $\mathrm{SD}$ & mean & SD & mean & SD \\
\hline 1 & $\mathrm{AUC}_{\text {total }}[\mathrm{au}]$ & 89 & 60 & 94 & 61 & 19000 & 21000 & 27000 & 32000 & 270 & 180 & 300 & 200 & 2400 & 1800 & 3500 & 3400 \\
\hline 2 & Area $_{\max }[\mathrm{au}]$ & 24 & 17 & 24 & 19 & 7300 & 6600 & 9600 & 11000 & 93 & 86 & 120 & 130 & 450 & 310 & 1100 & 1400 \\
\hline 3 & $\mathrm{AUC}_{\text {pre }}[\mathrm{au}]$ & 7 & 8 & 7 & 8 & 3600 & 5600 & 7500 & 9700 & 11 & 14 & 18 & 21 & 88 & 135 & 1200 & 1500 \\
\hline 4 & Area $_{\text {max _pre }}[\mathrm{au}]$ & 15 & 24 & 16 & 25 & 8000 & 16000 & 15000 & 26000 & 23 & 35 & 36 & 50 & 216 & 370 & 1900 & 2600 \\
\hline 5 & $\mathrm{AUC}_{\text {post }}[\mathrm{au}]$ & 75 & 54 & 78 & 52 & 11000 & 11000 & 12000 & 13000 & 250 & 180 & 270 & 190 & 2200 & 1700 & 1600 & 1600 \\
\hline 6 & $T_{20+}[\mathrm{s}]$ & 40 & 12 & 40 & 14 & 29 & 11 & 28 & 11 & 37 & 10 & 35 & 9 & 53 & 17 & 31 & 15 \\
\hline 7 & $T_{20-}[\mathrm{s}]$ & 17 & 8 & 17 & 9 & 17 & 10 & 16 & 10 & 18 & 7 & 17 & 7 & 18 & 10 & 17 & 14 \\
\hline 8 & $\Delta T_{20}[\mathrm{~s}]$ & 22 & 10 & 23 & 12 & 12 & 5 & 11 & 4 & 20 & 8 & 17 & 7 & 35 & 16 & 14 & 7 \\
\hline 9 & $T_{\max }[\mathrm{s}]$ & 21 & 9 & 21 & 11 & 18 & 14 & 16 & 14 & 22 & 7 & 20 & 8 & 26 & 16 & 15 & 16 \\
\hline 10 & $C_{\max }[\mathrm{au}]$ & 25 & 15 & 25 & 15 & 12000 & 11000 & 20000 & 18000 & 72 & 53 & 95 & 83 & 390 & 260 & 2700 & 2600 \\
\hline
\end{tabular}

(Supplementary Figure 1), panelists, and products. Differences in signal intensity are evident with the most abundant ion from ethyl butyrate $(m / z 117$, Supplementary Figure 1C) and the least abundant ion from acetoin $(m / z$ 87, Supplementary Figure 1B).

During preprocessing with our procedure the real-time profiles are transformed into breath-by-breath profiles (as described in the sections Procedure and Methods).

Examples of standardized breath-by-breath profiles of all four monitored ions are presented in Supplementary Figure 2 (for panelist $\mathrm{p} 1$ with product $f f$ following protocol A). Already after this stage of preprocessing, direct observations can be made on the release profiles of the monitored compounds. For example, it can be seen that retro-nasal release of all monitored compounds occurs mainly after the first swallow in the fourth breath. After the fourth breath, an exponential decrease in compound intensities can be observed. For ethyl butyrate and ethyl octanoate a significant amount of the compound is also present in a period before the first swallow, in the first breath.

In the final step of preprocessing, 10 parameters, describing release kinetics, are successfully derived (Figure 2). These parameters are used during assessment of the panel performance. For example, Table 2 presents the mean levels and standard deviations of the kinetic parameters for the four compounds in the two products: $f f$ and ss including all protocols. The mean levels of kinetic parameters (e.g., $\left.\mathrm{AUC}_{\text {total }}\right)$ are in a good agreement with the observations made based on the breath-by-breath profiles (Supplementary Figure 2) and the real-time profiles (Supplementary Figure 1). A large spread of values is observed over the four protocols, evidenced by relatively large standard deviations. This variation is greatly reduced when the data is analyzed per protocol. Nevertheless, slight differences between the products $f f$ and $s s$ can already be observed within the standard deviation, such as for the ions ethyl butyrate and ethyl octanoate and parameters $\mathrm{AUC}_{\text {total }}$, Area $_{\max \text { pre, }}$ and $\mathrm{AUC}_{\text {pre. }}$. Results of more detailed analysis of the differences between products and protocols are presented in the next paragraphs.

Panel Repeatability. First, standard ANOVA models are employed to assess panel repeatability and homogeneity. Repetition was not found to be a significant factor for any of these models. However, many significant interactions between panelists and protocols are found (see Supplementary Figure 3 ). This means that the panel is heterogeneous in relation to the experimental protocol. It is true especially for acetoin and anisaldehyde for which monitored ions are present in relatively low levels (see Table 2); as such, they are more sensitive to the characteristics of the experimental protocol.

From this univariate analysis, it can be concluded that panelists are performing differently in different protocols, providing different release profiles of monitored compounds. One protocol with the best repeatability should be selected for all panelists to avoid such interaction effects. Repeatability within each protocol and between different protocols is evaluated and described below.

Repeatability within Each Protocol. Grouping of the samples, coming from different ions of the same panelist, can be observed in PCA scatterplots (Supplementary Figures 4 and 5) for all products and protocols. The samples coming from panelist p6 are well separated from other samples in PC1 direction. Panelist p6 was identified as an outlier after further detailed inspection of the kinetic parameter values of this panelist, which showed to be very irreproducible. The data from panelist p6 is discarded from further analysis hereafter.

PCA was repeated for the data of the remaining seven panelists (Supplementary Figure 6 and 7). Similar grouping of the samples, coming from different ions of the same panelist, can be seen as when p6 was included, but without clear separations of panelists. Correlations can be observed between repeatability obtained for different kinetic parameters (PCA loading plots, results not shown). The individual panelist repeatability of $\mathrm{AUC}_{\text {total }}, \mathrm{Area}_{\max }, \mathrm{AUC}_{\text {post }}$ and $\mathrm{C}_{\max }$ are usually highly correlated (similar loading directions in PCA, first group of parameters) and responsible for the distribution of samples in PC1 (accounting for $41-49 \%$ of explained variance). Another group of correlated parameters (containing $T_{20+}$, $T_{20-}$, and $T_{\max }$, second group of parameters) contributes mainly to PC2 (accounting for $16-21 \%$ of explained variance). A third group of parameters, comprising $\mathrm{Area}_{\max }$ pre and $\mathrm{AUC}_{\text {pre }}$, as well as stand-alone $\Delta T_{20}$, are contributing both to PC1 and PC2.

Repeatability between Different Protocols. For both products, some grouping of samples from the same panelist can be seen when score plots of PC1 vs PC2 for product $f f$ and PC1 vs PC3 for product ss are studied (Supplementary Figure 8). Repeatability of kinetic parameters in protocol A (a reference protocol) is the main contributor to PC1 direction (accounting for $21 \%$ for product $f f$ and $33 \%$ for product $s$ ). Repeatability of the individual panelist in protocol A is lower than for other protocols, especially for product ss. No clear trends in differences between repeatability of kinetic parameters in other protocols: B, C, and D can be seen. 
Figure 2 and Supplementary Figure 9 show the pooled variance of protocols $\mathrm{B}, \mathrm{C}$, and D standardized to the pooled variance of the reference protocol $\mathrm{A}$. The panel's repeatability in the protocols B, C, and D is significantly better (see Supplementary Table 1) than in protocol A for both products. However, the repeatability of the kinetic parameters $T_{20}$ and $T_{\max }$ in protocol $\mathrm{D}$, for product $f f$ with ethyl butyrate $(\mathrm{m} / \mathrm{z}$ $117)$ and with ethyl octanoate $(m / z 173)$ is much lower (up to 12 -fold) than in other protocols (Supplementary Figure 9D and 9F). This is mostly related to the multiple swallows in protocol $\mathrm{D}$, which cause additional release peaks of ethyl butyrate and ethyl octanoate. The intensity of these peaks is fluctuating, resulting in high variability in determining $T_{\max }$ and $T_{20-}$.

For product ss, the strongest improvement in panel repeatability can be observed for the most controlled protocol, protocol C (Figure 3 and Supplementary Figure 9A,B),

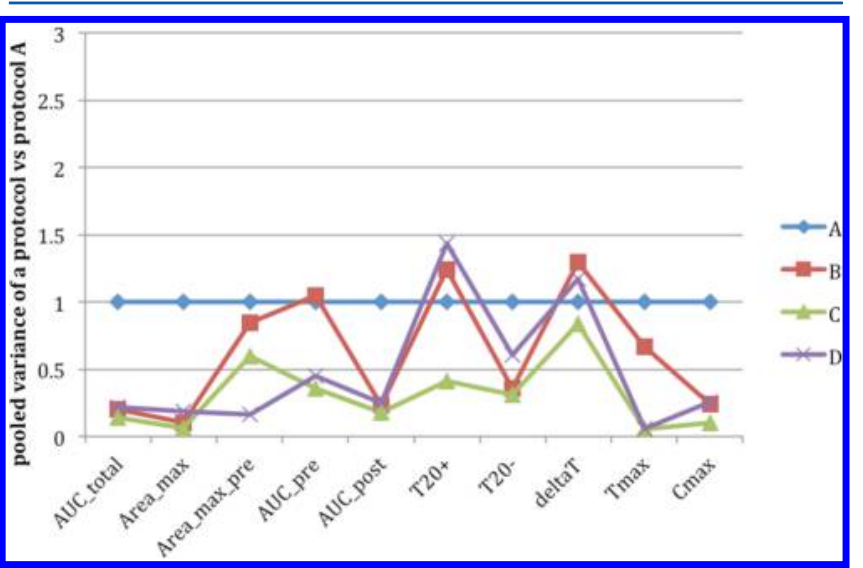

Figure 3. Pooled variance of the panel for four experimental protocols: A, B, C, and D for anisaldehyde and product ss. Pooled variance of protocol A is taken as a reference, and values of pooled variance of all protocols are normalized to that of protocol A.

especially for parameters relating to release before swallowing. Similarly to product $f f$, decrease in repeatability of $T_{20-}$ and $T_{\max }$ for ethyl butyrate and ethyl octanoate, is present. However, it is observed on a smaller scale and includes acetoin as well. This can be explained by a lack of lipophilic phase in the matrix of product $s s$ and is further discussed in the Discussion section.

When the observed differences between the protocols are compared between products, lower significance levels are observed for product ss (Supplementary Table 1). For product ss, protocol $\mathrm{C}$ is clearly the protocol with the highest panel repeatability. It is significantly better in comparison with all protocols (including protocol $\mathrm{B}$ and $\mathrm{D}$ ).

Discrimination Power of Different Protocols. According to the univariate analysis results (Supplementary Figure 10), it cannot be concluded which protocol is characterized with the greatest discrimination power. Differences between products are both protocol- and compound-dependent. No differences between products can be observed for acetoin. In the case of ethyl octanoate, many significant differences can be reported. These are mainly related to increased levels of Area $\mathrm{max}_{\text {pre }}$ $\mathrm{AUC}_{\text {pre }}$ and $C_{\max }$ and decreased levels of $T_{20+}, \Delta T_{20}$, and $T_{\max }$ in product ss when compared with ff (see Table 2). For ethyl butyrate, significant differences are observed in protocol $\mathrm{B}$ and $\mathrm{C}$ for Area $_{\max }$ pre, $\mathrm{AUC}_{\text {pres }}$ and $C_{\max }$. These parameters have significantly higher levels for product $s s$ when compared with product $f f$, in line with an expected quicker release from nonfatcontaining matrices leading to higher intensities in the earlier part of the release curve. For anisaldehyde, significant differences are observed for protocols $\mathrm{A}$ and $\mathrm{D}$ and include different parameters (protocol A: $\mathrm{AUC}_{\text {total, }}$ Area $_{\max }, \mathrm{AUC}_{\text {post }}$ $C_{\max }$, protocol D: Area $\left.\max _{\max }, \Delta T_{20}, T_{\max }, C_{\max }\right)$. Significant parameters in protocol A have higher values in product $s s$ than in product $f f$. Area $\max _{\text {pre }}$ and $C_{\max }$ have significantly higher values in product $s s$ than $f f$ in protocol $\mathrm{D}$. In contrast, $\Delta T_{20}$ and $T_{\max }$ have lower values in product $s s$ than $f f$ in protocol $\mathrm{D}$, again in line with expectation.

Results of multivariate analysis of discrimination power of different protocols are presented in Table 3. It can be observed

Table 3. Results of Multilevel-PLS-DA Modeling of Differences between Product $f f$ and ss in Four Protocols

\begin{tabular}{|c|c|c|c|}
\hline \multirow[b]{2}{*}{ protocol } & \multicolumn{2}{|c|}{ model performance } & \multirow[b]{2}{*}{ significant variables ${ }^{c}$ ( $p$-values) } \\
\hline & $\overline{\mathrm{NMC}^{a}(\%)}$ & $\operatorname{AUROC}^{b}$ & \\
\hline A & 16.3 & 0.91 & $1(0.008), 2(0.002), 3(0.0325)$ \\
\hline B & 11.8 & 0.94 & $\begin{array}{c}1(0.0005), 2(0.0045), 3(0.0255) \\
4(0.0405)\end{array}$ \\
\hline $\mathrm{C}$ & 8.9 & 0.96 & $1(0.0005), 2(0.008)$ \\
\hline $\mathrm{D}$ & 4.0 & 0.99 & $1(0.01), 2(0.0035), 5(0.0425)$ \\
\hline
\end{tabular}

${ }^{a} \mathrm{NMC}(\%)-\%$ of misclassified samples. ${ }^{b}$ AUROC - area under receiver operating curve. ${ }^{c}$ Significant variables: $1-\mathrm{i} 173 T_{20+}, 2-\mathrm{i} 173$ $\Delta T_{20}, 3-\mathrm{i} 173 \_C_{\max }$ 4-i173_ $\mathrm{AUC}_{\text {pre }}, 5-\mathrm{i} 173$ _ $\mathrm{AUC}_{\text {post }}$

that all protocols are characterized as having a very good discrimination power ( $\mathrm{NMC} \%<17 \%$ and AUROC > 0.91). The best model performance is obtained for protocol $\mathrm{D}$. The second best is protocol $\mathrm{C}$. The variables important for discrimination of products include mainly kinetic parameters of ethyl octanoate confirming findings of univariate analysis. These are i173_ $T_{20+}$, i173_ $\Delta T_{20}, 3-\mathrm{i} 173 \_C_{\max }$, and

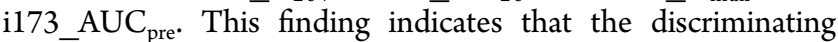
power of each protocol is mainly dependent on the kinetic parameters of ethyl octanoate; the release of other compounds from the two products is similar. This is expected, because the release of the highly lipophilic ethyl octanoate will be considerably more influenced than that of the other compounds by the presence of an oil phase, which functions as a sink.

\section{DISCUSSION}

Developed Procedure. In our procedure, the breathing pattern indicated by acetone (reference ion) is used to transform unsynchronized time profiles of the monitored ions into synchronized breath profiles. According to our knowledge, this is the most accurate preprocessing approach available to obtain release profiles. Other approaches are much less exact, such as smoothing of the time profile ${ }^{16}$ or manually taking the maximum intensity of breath peaks. ${ }^{14}$ In addition, incorporating the normalization of breath profiles by a blank period corrects for differences between baseline levels in different panelists. That makes further comparison between panelists more relevant.

The presented preprocessing approach is fully automated and user-independent. The user input is limited to the loading of the PTR-MS data files and a file with starting, product and swallow times. The complete data preprocessing of the 318 assays took less than $10 \mathrm{~min}$ of computational time. This is a substantial improvement when compared with manual 
integration of breath patterns (for such a data set, typically a 2 week period).

A wide spectrum of kinetic parameters is provided with our procedure and included in this study. It should be emphasized that the derived parameters $T_{\max }, T_{20+}, T_{20-}$, and $\Delta T_{20}$ provide additional information on release profiles (such as aroma persistence), available only after data preprocessing. This information can be used, for example, to detect irreproducible experimental protocols (see the section Comparisons of Four Experimental Protocols). In our procedure, a release profile can be automatically divided into a pre- and postswallowing period, and the kinetic parameters can be more accurately described for each period. $^{22}$

In the final step of the presented procedure, univariate and multivariate tools are applied to assess differences between products and experimental protocols. Multivariate analysis became possible due to the availability of many parameters derived with the preprocessing procedure. Therefore, PCA is employed to study individual panelist repeatabilities for the first time. Moreover, ML-PLS-DA is used to detect product differences in in vivo release studies, also for the first time. It should be stressed that including these multivariate tools enabled identification of crossover effects between product composition and experimental protocol. This was not possible with only standard univariate tools such as ANOVA. Nevertheless, the used combination of methods leads to valuable insights about changes in retro-nasal release of the four studied compounds in the two products. Aforementioned outcomes are discussed below.

Comparisons of Four Experimental Protocols. The extent of retronasal aroma release is a physiological feature that can be individually characterized. ${ }^{28}$ Subject differences in oral processing parameters, such as salivary flow rate, nasal anatomy, bite size, and eating speed are (partly) responsible for this. Bite size and duration of oral processing can be controlled in an experimental protocol by a standardized amount of product taken, fixed time of mastication before the first swallow and secondary swallows. ${ }^{14,16,29}$ This was the case in our study, in which three levels of control were implemented: a free protocol with free time to the first swallow (protocol A, a reference protocol), protocols with fixed time $(20 \mathrm{~s})$ to the first swallow (protocols B and C), and a protocol with a fixed time to first and three secondary swallows (every $20 \mathrm{~s}$, protocol D). The additional control over breathing pattern was introduced in protocol $\mathrm{C}$, and this may influence repeatability of time profiles, especially for untrained panelists.

As expected and shown in this study, the panel is heterogeneous in relation to the experimental protocol. Each panelist has different levels of individual repeatability dependent on experimental protocol and considered kinetic parameters. The latter are grouped and correspond mainly to two independent aspects of aroma release: initial release of volatiles including first swallow (1st group: $\mathrm{AUC}_{\text {total }}$, Area $_{\max }, \mathrm{AUC}_{\text {post }}$, and $C_{\max }$ ) and the persistence of volatiles in breath (2nd group: $T_{20+}, T_{20-}$, and $\left.T_{\max }\right)$. This is in agreement with the findings of Hodgson et al. ${ }^{14}$ where influence of initial swallow and secondary swallows was studied. Additionally, it gives a new insight into the relationships between kinetic parameters and the differences observed for different protocols and panelists.

For both products, panel repeatability of protocols B, C, and $\mathrm{D}$ was better than the reference protocol. The most controlled protocol C was characterized with the best repeatability; compared with all other protocols, the improvement in repeatability was statistically significant for aqueous product ss. Protocol D gave very irreproducible results for $T_{20-}$ and $T_{\max }$ for the esters ethyl butyrate and ethyl octanoate, especially for the full-fat milk matrix product, ff. Taking this into account, we do not recommend using this protocol for studying the release of these lipophilic compounds, at least not in cases where time parameters are in focus.

The significant improvement in discrimination power for protocols $\mathrm{B}, \mathrm{C}$, and $\mathrm{D}$ in comparison to free protocol $\mathrm{A}$ is reported. This is in contrast to previously reported studies where a free protocol had the highest discriminative ability. ${ }^{22}$ The most discriminative kinetic parameters were derived for ethyl octanoate. For this compound, increased levels of Area $_{\text {max pre, }} \mathrm{AUC}_{\text {pre, }}$ and $C_{\max }$ and decreased levels of $T_{20+}$, $\Delta T_{20}$, and $T_{\max }$ are reported in products with the aqueous sugar solution matrix when compared with the full-fat milk matrix. This is perfectly in line with the expected slower release of lipophilic compounds from oil-containing matrices.

In the preswallow phase, the effect of food composition is expected to have the greatest effect on volatile release. In the postswallow phase, where most of the product is removed, differences in the release are mainly attributed to properties of the compounds (i.e., volatile partitioning in the fat, in the bolus, and in masticated particles coating the mouth and throat). ${ }^{16}$ Results reported in this paper strictly correspond to the above statements. The increase in $A_{\text {rea }}$ max pre and $\mathrm{AUC}_{\text {pre }}$ for the product with sugar solution matrix is observed for all compounds and is statistically relevant for ethyl octanoate (for all protocols) and ethyl butyrate (for the protocols B and C). Both esters, which are highly volatile (high $K_{\mathrm{a} / \mathrm{w}}$, see Table $1)$, are released to retronasal space more easily pre- and postswallow than the two other considered compounds. Ethyl octanoate has the strongest persistence after swallow (high $T_{20+}$ and $\Delta T_{20}$ values) when compared with other compounds (Table 2) because it is highly lipophilic (high $\log P$ ) and stays longer in the fat in the bolus of the $f f$ product.

\section{CONCLUSIONS}

A comprehensive data analysis procedure was developed and employed for the analysis of a large data set from the real-time, in vivo aroma release study, using PTR-MS. It comprises an automated data preprocessing procedure and multivariate assessment of the panel performance. During preprocessing, real-time breath profiles are converted into standardized breath profiles and 10 kinetic parameters are derived and used to describe the release kinetics of the aroma compounds. Next to this, multivariate analysis enabled extracting and interpreting complementary information about panel performance, individual panelists, studied products, aroma compounds, and kinetic parameters.

The most controlled experimental protocol with one swallow event and a controlled breathing pattern was selected as the optimal one and is recommended in other in vivo release studies.

\section{ASSOCIATED CONTENT}

\section{S Supporting Information}

The Supporting Information is available free of charge on the ACS Publications website at DOI: 10.1021/acs.analchem.5b02420.

Experimental details and supplemental data, as noted in the text (PDF) 


\section{AUTHOR INFORMATION}

\section{Corresponding Author}

*E-mail: chemometrics@science.ru.nl.

\section{Notes}

The authors declare no competing financial interest.

\section{ACKNOWLEDGMENTS}

This research received funding from The Netherlands Organization for Scientific Research (NWO) in the framework of the Technology Area COAST; project: Breath volatiles; 053.21.103. Ewa Szymańska would like to thank Anouk Suppers for optimizing parameters of the baseline correction method.

\section{REFERENCES}

(1) Cao, W.; Duan, Y. Crit. Rev. Anal. Chem. 2007, 37, 3-13.

(2) Trefz, P.; Schmidt, M.; Oertel, P.; Obermeier, J.; Brock, B.; Kamysek, S.; Dunkl, J. r.; Zimmermann, R.; Schubert, J. K.; Miekisch, W. Anal. Chem. 2013, 85, 10321-10329.

(3) Mayr, D.; Märk, T.; Lindinger, W.; Brevard, H.; Yeretzian, C. Int. J. Mass Spectrom. 2003, 223-224, 743-756.

(4) Samudrala, D.; Geurts, B.; Brown, P. A.; Szymańska, E.; Mandon, J.; Jansen, J.; Buydens, L. M.; Harren, F. J. M.; Cristescu, S. M. Metabolomics 2015, in press, DOI: 10.1007/s11306-015-0813-8.

(5) Linforth, R.; Taylor, A. J. I. Agric. Food Chem. 2000, 48, 54195423.

(6) Haahr, A.-M.; Madsen, H.; Smedsgaard, J.; Bredie, W. L.; Stahnke, L. H.; Refsgaard, H. H. Anal. Chem. 2003, 75, 655-662.

(7) Linforth, R. S.; Blissett, A.; Taylor, A. J. J. Agric. Food Chem. 2005, $53,7217-7221$.

(8) Rabe, S.; Linforth, R. S.; Krings, U.; Taylor, A. J.; Berger, R. G. Chemical senses 2004, 29, 163-173.

(9) Beauchamp, J.; Kirsch, F.; Buettner, A. L. Breath Res. 2010, 4, 026006.

(10) Luchner, M.; Gutmann, R.; Bayer, K.; Dunkl, J.; Hansel, A.; Herbig, J.; Singer, W.; Strobl, F.; Winkler, K.; Striedner, G. Biotechnol. Bioeng. 2012, 109, 3059-3069.

(11) Smilde, A.; Westerhuis, J.; Hoefsloot, H.; Bijlsma, S.; Rubingh, C.; Vis, D.; Jellema, R.; Pijl, H.; Roelfsema, F.; van der Greef, J. Metabolomics 2010, 6, 3-17.

(12) Smit, S.; Szymańska, E.; Kunz, I.; Roldan, V. G.; van Tilborg, M. W.; Weber, P.; Prudence, K.; van der Kloet, F. M.; van Duynhoven, J. P.; Smilde, A. K.; de Vos, R. C. H.; Bendik, I. Mol. Nutr. Food Res. 2014, 58, 2111-2121.

(13) Van Duynhoven, J.; Van Velzen, E.; Westerhuis, J.; Foltz, M.; Jacobs, D.; Smilde, A. Trends Food Sci. Technol. 2012, 26, 4-13.

(14) Hodgson, M.; Langridge, J.; Linforth, R.; Taylor, A. J. Agric. Food Chem. 2005, 53, 1700-1706.

(15) Mei, J. B.; Reineccius, G. A.; Knighton, W. B.; Grimsrud, E. P. J. Agric. Food Chem. 2004, 52, 6267-6270.

(16) Frank, D. C.; Eyres, G. T.; Piyasiri, U.; Delahunty, C. M. Flavour Fragrance I. 2012, 27, 433-444.

(17) Ruijschop, R. M.; Burgering, M. J.; Jacobs, M. A.; Boelrijk, A. E. Chem. Senses 2009, 34, 395-403.

(18) Buszewski, B.; Kęsy, M.; Ligor, T.; Amann, A. Biomed. Chromatogr. 2007, 21, 553-566.

(19) Rossi, F. Food quality and preference 2001, 12, 467-479.

(20) Eilers, P. H. C. Anal. Chem. 2003, 75, 3631-3636.

(21) Vargas Aguilera, C. A. http://www.mathworks.com/ matlabcentral/fileexchange/12275-extrema-m--extrema2-m (assessed February 20, 2015).

(22) Mesurolle, J.; Saint-Eve, A.; Déléris, I.; Souchon, I. Molecules 2013, 18, 6035-6056.

(23) Mandel, J. Chemom. Intell. Lab. Svst. 1991, 11, 109-119.

(24) Benjamini, Y.; Hochberg, Y. Journal of the Royal Statistical Society. Series B (Methodological) 1995, 289-300.
(25) van Velzen, E. J.; Westerhuis, J. A.; van Duynhoven, J. P.; van Dorsten, F. A.; Hoefsloot, H. C.; Jacobs, D. M.; Smit, S.; Draijer, R.; Kroner, C. I.; Smilde, A. K. I. Proteome Res. 2008, 7, 4483-4491.

(26) Szymańska, E.; van Dorsten, F. A.; Troost, J.; Paliukhovich, I.; van Velzen, E. J.; Hendriks, M. M.; Trautwein, E. A.; van Duynhoven, J. P.; Vreeken, R. J.; Smilde, A. K. Metabolomics 2012, 8, 894-906.

(27) Szymańska, E.; Saccenti, E.; Smilde, A.; Westerhuis, J. Metabolomics 2012, 8, 3-16.

(28) Ruijschop, R. M.; Boelrijk, A. E.; de Graaf, C.; WesterterpPlantenga, M. S. I. Agric. Food Chem. 2009, 57, 9888-9894.

(29) Hodgson, M.; Linforth, R.; Taylor, A. J. Agric. Food Chem. 2003, $51,5052-5057$. 